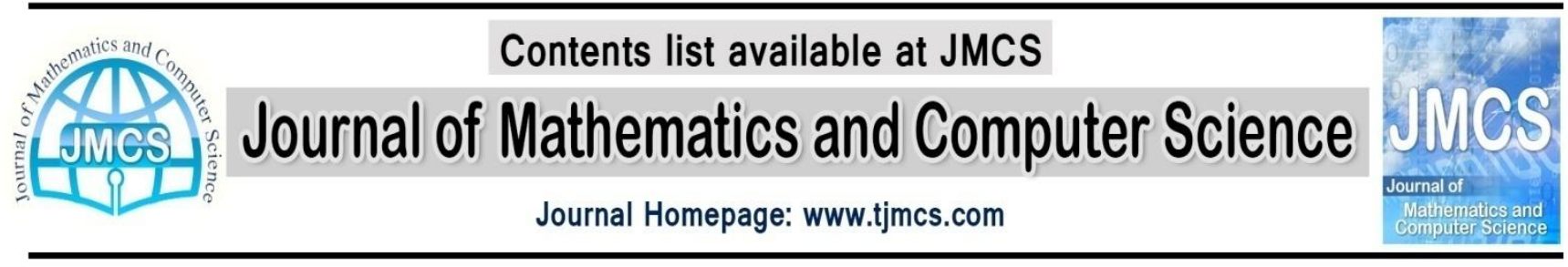

\title{
Arzela-Ascoli theorem for Felbin's type fuzzy normed linear spaces
}

\author{
A. Zohourmeskar, A. Mozaffarikhan \\ Department of basic science, Jundi-shapur university of Technology, Dezful, Iran, \\ First University. \\ Department of Mathematics, Mahshahr Branch, Islamic Azad University, Mahshahr,Iran. \\ zohourmeskar@yahoo.com,mozaffarikhan.a@gmail.com
}

Article history:

Received July 2014

Accepted September 2014

Available online September 2014

\begin{abstract}
In this note, we define the strongly fuzzy bounded linear operators on Felbin's fuzzy normed linear space. In particular, a Arzela-Ascoli theorem for strongly fuzzy bounded linear operators are established.
\end{abstract}

Key words and phrases: Fuzzy real number, Fuzzy norm linear space, Strongly fuzzy continuous, Strongly fuzzy bounded.

\section{Introduction}

In 1992, Felbin[3], by the notion of a fuzzy metric space introduced the concept of a fuzzy normed linear space. In fact she did this by assigning a non-negative fuzzy real number to each element of a linear space. Bag and Samanta [1] gave the definitions of strongly fuzzy bounded linear operators. They proved that both the set $\mathrm{B}(\mathrm{X}, \mathrm{Y})$ sof all strongly fuzzy bounded linear operators from a Felbin's type fuzzy normed linear space $X$ into another Felbin's type fuzzy normed space $Y$ are fuzzy normed linear spaces. A Arzela-Ascoli's theorem for fuzzy strongly bounded linear operators were established in this work. In this paper we consider the recent definition of Bag and Samanta for fuzzy bounded linear operators. In section 2, we state some preliminaries and concepts, whih are needed for our study. In section 3, we try to make a version of Arzela-Ascoli theorem in a fuzzy normed linear space.

\section{Preliminaries}

We denote the set of all real number by $\mathbb{R}$ and by $\mathbb{Z}^{+}$the set of all positive integers. 
In this paper, we consider the concept of fuzzy real number in the sense Xiao and Zhu [5] which is defined below:

A mapping $\eta(t): \mathbb{R} \rightarrow[0,1]$, whose $\alpha$-level set is denoted by $[\eta]_{\alpha}=\{t: \eta(t) \geq \alpha\}$, is called a fuzzy real number if it satisfies two axioms:

(N1) There exists $t_{0} \in \mathbb{R}$ such that $\eta\left(t_{0}\right)=1$;

(N2) For each $\alpha \epsilon(0,1] ;[\eta]_{\alpha}=\left[\eta_{\alpha}^{-}, \eta_{\alpha}^{+}\right]$, where $-\infty<\eta_{\alpha}^{-} \leq \eta_{\alpha}^{+}<+\infty$.

The set of all fuzzy real number is denoted by $\mathbb{F}$. For each $r \in \mathbb{R}$, let $\bar{r} \in \mathbb{F}$ be defined by

$$
\bar{r}(t)= \begin{cases}1 & t=r \\ 0 & t \neq r\end{cases}
$$

So $\bar{r}$ is a fuzzy real number and $\mathbb{R}$ can be embedded in $\mathbb{F}$. Let $\eta \in \mathbb{F}, \eta$ is called positive fuzzy real number if for all $t<0, \eta(t)=0$. The set of all positive fuzzy real numbers is denoted by $\mathbb{F}^{+}$. A partial order ' $\leqslant$ ' in $\mathbb{F}$ is defined as follows, $\eta \leqslant \delta$ if and only if for all $\alpha \epsilon(0,1], \eta_{\alpha}^{-} \leq \delta_{\alpha}^{-}$and $\eta_{\alpha}^{+} \leq \delta_{\alpha}^{+}$where, $[\eta]_{\alpha}=\left[\eta_{\alpha}^{-}, \eta_{\alpha}^{+}\right]$and $[\delta]_{\alpha}=\left[\delta_{\alpha}^{-}, \delta_{\alpha}^{+}\right]$. The strict inequality in $\mathbb{F}$ is defined by $\eta<\delta$ if and only if for all $\alpha \epsilon(0,1], \eta_{\alpha}^{-}<\delta_{\alpha}^{-}$and $\eta_{\alpha}^{+}<\delta_{\alpha}^{+}$. According to Dubois and Prade[2], the airthmetic operations $\oplus, \ominus, \odot, \oslash$ on $\mathbb{F} \times \mathbb{F}$ are defined by

$$
\begin{array}{ll}
(\eta \bigoplus \delta)(t)=\sup _{t=r+s} \min \{\eta(r), \delta(s)\}, & t \in \mathbb{R} \\
(\eta \ominus \delta)(t)=\sup _{t=r-s} \min \{\eta(r), \delta(s)\}, & t \in \mathbb{R} \\
(\eta \odot \delta)(t)=\sup _{t=r s} \min \{\eta(r), \delta(s)\}, & t \in \mathbb{R} \\
(\eta \oslash \delta)(t)=\sup _{t=\frac{r}{s}} \min \{\eta(r), \delta(s)\}, & t \in \mathbb{R}
\end{array}
$$

Definition 2.1[1,Definition 2.4] Let $X$ be a linear space over $\mathbb{R}$. Suppose $\|\|:. X \rightarrow \mathbb{F}^{+}$is a mapping satisfying

And

$$
\begin{gathered}
\text { (i) }\|x\|=\overline{0} \text { if and only if } x=0, \\
\text { (ii) }\|r x\|=|r|\|x\|, x \in X, r \in R, \\
\text { (iii)for all } x, y \in X,\|x+y\| \leqslant\|x\| \oplus\|y\|
\end{gathered}
$$

$$
\left(A^{\prime}\right): x \neq 0 \Rightarrow\|x\|(t)=0, \forall t \leq 0 .
$$

$(X,\|\|$.$) is called a fuzzy normed linear space and \|$.$\| is called a fuzzy norm on X$. In the rest of this paper we use the previous definition of fuzzy norm. We note that

(i)condition $\left(A^{\prime}\right)$ in Definition 2.1 is equivalent to the condition

$\left(A^{\prime \prime}\right)$ : For all $x(\neq 0) \in X$ and each $\alpha \in(0,1],\|x\|_{\alpha}^{-}>0$, where $[\|x\|]_{\alpha}=\left[\|x\|_{\alpha}^{-},\|x\|_{\alpha}^{+}\right]$and (ii) $\|x\|_{\alpha}^{i}, i=1,2$ are crisp norms on $X$.

According to J. Xiao and $X$. Zhu [5], let $(X,\|\|$.$) be an fuzzy normed linear space, A \subseteq X$ and $x_{0} \in X . x_{0}$ is called a point of closure of $A$ if $\left\{x_{0}+N(\alpha, \alpha)\right\} \cap A \neq \phi$ for every $\alpha \in(0,1] ; \bar{A}$ denotes the set of all points of closure of $A$. Ais called a fuzzy closed set if $A=\bar{A}$. Ais called separable if there is a countable set $B$ such that $A \subseteq \bar{B}$. Ais called a fuzzy bounded set if for each $\alpha \in(0,1]$ there exists $M=M(\alpha)>0$ such that $A \subset N(M, \alpha)$. Ais called precompact if for each $\alpha \in(0,1]$ there exists a finite subset $\left\{x_{\alpha_{1}}, x_{\alpha_{2}}, \ldots, x_{\alpha_{n}}\right\} \subseteq X$ such that $A \subseteq U_{k=1}^{n}\left(x_{\alpha_{k}}+N(\alpha, \alpha)\right)$.

A sequence $\left\{x_{n}\right\}$ in $X$ is said converge to $x \in X$ if and only if for each $\alpha \in(0,1], \lim _{n \rightarrow \infty} \| x_{n}-$ $x \alpha+=0$. In this case we write limn $\rightarrow \infty x n=x$. Also a sequence $x n$ is called a Cauchy sequence if for each $\alpha \in(0,1], \lim _{m, n \rightarrow \infty}\left\|x_{n}-x_{m}\right\|_{\alpha}^{+}=0$. A fuzzy normed linear space $(X,\|\|$.$) is said to be$ complete if every Cauchy sequence in $X$ converges in X. Using theorem 4.3 and theorem 4.4 in [5], we have

(1) A is compact if and only if $A$ is precompact and complete.

(2) A is precompact, then $A$ is separable. 
Lemma 2.2 [6, Lemma 3.1] Let $(X,\|\|$.$) be a fuzzy normed linear space. \lim _{n \rightarrow \infty} x_{n}=x$ if and only if for every $\alpha \in(0,1]$ there is $K=K(\alpha) \in \mathbb{Z}^{+}$such that $\left\|x_{n}-x\right\|_{\alpha}^{+} \leq \alpha$ for all $n \geq K$.

A family $\left\{f_{n}\right\}$ of functions from an fuzzy normed space $X$ to a fuzzy normed space $Y$ is said to be equicontinunus if for every $\varepsilon \in \mathbb{F}^{+}$, there exist $\delta \in \mathbb{F}^{+}$such that for all $x, y \in X,\|x-y\| \prec \delta$ implies $\left\|f_{n}(x)-f_{n}(y)\right\| \prec \varepsilon$ for all $n \in N$. A sequence $\left\{f_{n}\right\}$ of functions from $X$ to $Y$ is said to be converge uniformly to a function $f$ from $X$ to $Y$ if for each $\alpha \in(0,1]$, there exist $K \in \mathbb{Z}^{+}$such that $\left\|f_{n}(x)-f(x)\right\|_{\alpha}^{+} \leq \alpha$ for all $n \geq K$ and for all $x \in X$.

Let $(X,\|\|$.$) and (Y,\|\|$.$) be two fuzzy normed linear spaces. A function T: X \rightarrow Y$ is said to be strongly fuzzy continuous at $x_{0} \in X$ if for a given $\varepsilon>0, \exists \delta>0$ such that

$\left\|T x-T x_{0}\right\|_{\alpha}^{+}<\varepsilon$ whenever $\left\|x-x_{0}\right\|_{\alpha}^{-}<\delta$

Where for $\alpha \epsilon(0,1],[\delta]_{\alpha}=\left[\delta_{\alpha}^{-}, \delta_{\alpha}^{+}\right]$.

Also a linear mapping $T: X \rightarrow Y$ is called strongly fuzzy bounded, if there exists a real number $K>0$ such that for each $x(\neq 0) \epsilon X,\|T x\| \oslash\|x\| \preccurlyeq K$. In this case, the set of all strongly fuzzy bounded linear operators defined from $X$ to $Y$ is denoted by $B(X, Y)$ (see [1]).

From [4] we know that a linear mapping $T: X \rightarrow Y$ is strongly fuzzy continuous if and only if it is strongly fuzzy bounded. Also the set $B(X, Y)$ is a linear space with respect to usual operations.

\section{The main results}

In this section, we will show a versoin of Arzela-Ascoli theorem for Felbin's type fuzzy normed.

Theorem 3.1 Let $\left\{f_{n}\right\}$ is a sequence of stongly fuzzy bounded linear operator over countable set $E$, then $\left\{f_{n}\right\}$ has subsequence $\left\{f_{n_{k}}\right\}$ that $\left\{f_{n_{k}}(x)\right\}$ for every $x \in E$ is convergent.

Proof.By Similar argument to that of classic case.

Lemma 3.2Let $\left\{f_{n}\right\}$ be an equicontinuous sequence of function from an fuzzy normed spaceX to a complete fuzzy normed space $Y$. If $\left\{f_{n}\right\}$ converges for each point of a dense subset Dof $X$. Then $\left\{f_{n}\right\}$ converges for each point of $X$.

Proof. Since $\left\{f_{n}\right\}$ is an equicontinuous family, for every $\varepsilon \in \mathbb{F}^{+}$there exists $\delta \in \mathbb{F}^{+}$such that for each $x, y \in X,\|x-y\| \prec \delta$ then $\left\|f_{n}(x)-f_{n}(y)\right\| \prec \varepsilon$. Suppose that $x \in X$ there exists $\alpha \in(0,1]$, such that $y \in D \cap\{x+N(\alpha, \alpha)\}$. $\left\{f_{n}(y)\right\}$ converges for that $y$. Since $\left\{f_{n}(y)\right\}$ is a Cauchy sequence, there exists $n_{0} \in N$ such that $\lim _{n, m \rightarrow \infty}\left\|f_{n}(x)-f_{n}(y)\right\|=0$, for all $m, n \geq n_{0}$. Now for any $x \in X$, we have

$\lim _{n, m \rightarrow \infty}\left\|f_{n}(x)-f_{m}(x)\right\| \preccurlyeq \lim _{n, m \rightarrow \infty}\left\|f_{n}(x)-f_{m}(y)\right\| \oplus \lim _{n, m \rightarrow \infty}\left\|f_{m}(y)-f_{n}(y)\right\| \oplus$ $\lim _{n, m \rightarrow \infty}\left\|f_{n}(y)-f_{m}(x)\right\|$. Hence $\left\{f_{n}(x)\right\}$ is a Cauchy sequence in $Y,\left\{f_{n}(x)\right\}$ converges.

Theorem 3.3 (Arzela-Ascoli theorem) Let $X$ be a fuzzy normed space and $Y$ is a fuzzy complete normed space and $K \subset X$, fuzzy compact if $\left\{f_{n}\right\},(n=1,2, \ldots)$ strongly fuzzy bounded linear operators and equicontunuous then there exists a strongly fuzzy bounded linear operator $f$ from $K$ to $Y$ and a subsequence $\left\{f_{n_{i}}\right\}$ of $\left\{f_{n}\right\}$ such that $\left\{f_{n_{i}}\right\}$ converges uniformly to $f$ on $K$. 
Proof. Let $E$ be a countable dense subset of K. Theorem 3.1, $\left\{f_{n}\right\}$ has a subsequence $\left\{f_{n_{i}}\right\}$ such that $\left\{f_{n_{i}}(x)\right\}$ for all $x \in E$ converges and by Lemma 3.2, converges for each point of $K$. Let $f(x)=\lim _{n \rightarrow \infty} f_{n_{i}}(x)$. We claim that $f$ is strongly fuzzy bounded linear operator. Let $\varepsilon>0$ and $\delta=\frac{\varepsilon}{2 K}$. Also $\left\|x-x_{0}\right\|_{\alpha}^{-}<\delta$ for all $\alpha \epsilon(0,1]$. Now for all $\alpha \epsilon(0,1]$, we have

$$
\left\|f(x)-f\left(x_{0}\right)\right\|_{\alpha}^{+} \leq\left\|f(x)-f_{n_{i}}(x)\right\|_{\alpha}^{+}+\left\|f_{n_{i}}(x)-f_{n_{i}}\left(x_{0}\right)\right\|_{\alpha}^{+}+\left\|f_{n_{i}}\left(x_{0}\right)-f_{n_{i}}\left(x_{0}\right)\right\|_{\alpha}^{+}
$$

Clearly

$$
\left\|f(x)-f\left(x_{0}\right)\right\|_{\alpha}^{+} \leq \frac{\varepsilon}{4}+K\left\|x-x_{0}\right\|_{\alpha}^{-}+\frac{\varepsilon}{4} \leq K \cdot \frac{\varepsilon}{2 K}+\frac{\varepsilon}{2}=\varepsilon
$$

Hence $f$ is strongly fuzzy continuous. Now we claim that $\left\{f_{n_{i}}\right\}$ converges uniformly to fon $K$.

For each $x \in K$, we have

$\left\|f_{n_{i}}(x)-f(x)\right\|_{\alpha}^{+} \leq\left\|f_{n_{i}}(x)-f_{n_{i}}\left(x_{i}\right)\right\|_{\alpha}^{+}+\left\|f_{n_{i}}\left(x_{i}\right)-f\left(x_{i}\right)\right\|_{\alpha}^{+}+\left\|f\left(x_{i}\right)-f(x)\right\|_{\alpha}^{+}$.

Clearly $\lim _{n \rightarrow \infty}\left\|f_{n_{i}}(x)-f(x)\right\|_{\alpha}^{+}=0$. Hence $\left\{f_{n_{i}}\right\}$ converges uniformly to fon $K$.

\section{References}

[1] T. Bag and S.K. Samanta, Fuzzy bounded linear operators in Felbin's type fuzzy normed linear spaces, Fuzzy Sets and Systems 159 (2008), 685-707.

[2] D. Dobois and H. Prade, Operations on fuzzy numbers, International Journal of System 9 (1978) 613 626.

[3] C. Felbin, Finite dimensional fuzzy normed linear spaces, fuzzy Set and System, 48 (1992), 239-248.

[4] A. Taghavi and M. Mehdizadeh, Adjoint operator in fuzzy normed linear spaces, The Journal of Mathematics and Computer Science, 3 (2011), 453-458.

[5] J. Xiao, and X. Zhu, On linearly topological structure and property of fuzzy normed linear space, Fuzzy Sets and Systems 125 (2002), 153-161.

[6]J. Z. Xiao, Y. Lu, Condensing operators and topological degree theory in standard fuzzy normed space, fuzzy Set and Systems (2009) 1-17. 